\title{
Estratégias para o Ensino do Autocuidado de pacientes Cirúrgicos: Uma Revisão Integrativa da Literatura
}

\author{
Strategies for Teaching Self-Care of Surgical Patients: An Integrative Review of the Literature \\ Estrategias para la Enseñanza de Auto Cuidado de Pacientes Quirúrgicos: Una Revisión Integral \\ de la Literatura
}

Debora Viviane Stadler ${ }^{1}$, Anercy Tojeiro Giordani², Gabriela

Machado Ezaias Paulino ${ }^{3}$, Helena Megumi Sonobe ${ }^{4}$, Renata

Rodrigues Zanardo ${ }^{5}$, Maria Aparecida Valério ${ }^{6}$

\section{Resumo}

O ensino do autocuidado se destaca como importante estratégia para o enfrentamento das dificuldades que permeiam o processo de reabilitação pós-operatória, possibilitando que as pessoas cuidem de si mesmas subsidiadas por informações e recomendações elaboradas criteriosamente. Assim, este artigo teve como objetivo identificar e avaliar as evidências encontradas

\footnotetext{
${ }^{1}$ Graduada em Enfermagem-Bacharel pela Universidade Estadual do Norte do Paraná.

${ }^{2}$ Graduada em Enfermagem e Obstetrícia (1984) pela Faculdade de Medicina de Marília (FAMEMA), Mestrado (2000), Doutorado (2003) e Pós-doutorado (2013) pela Escola de Enfermagem de Ribeirão Preto da Universidade de São Paulo (EERPUSP).Especialista em Administração Hospitalar e em Enfermagem do Trabalho, com Aperfeiçoamento em Licenciatura Plena em Formação Pedagógica para Docentes. Professora Adjunta (nível D) do Curso de Enfermagem e docente do Programa de Pós-graduação (Mestrado Profissional em Ensino PPGEN)da Universidade Estadual do Norte do Paraná (UENP); Supervisora de Serviço e Vice Coordenadora(3a Gestão: 20162018)do Curso de Enfermagem da UENPCLM.Pesquisadora nas linhas: Educação em Saúde; Enfermagem e Humanização do cuidado, Doenças infecciosas: problemáticas e estratégias de enfrentamento e Formação de professores.

${ }^{3}$ Atua como Professora do Ensino Básico, Técnico e Tecnológico (EBTT) do Instituto Federal do Paraná - Campus Londrina. Doutorando em Enfermagem pela Universidade Estadual de Maringá. Professora Colaboradora/Assistente no Departamento de Enfermagem do Centro de Ciências da Saúde da Universidade Estadual de Londrina (2013-2015) e Enfermeira no Hospital Dr. Anísio Figueiredo da Secretaria de Saúde do Paraná em Londrina/PR (2010-2016). Graduada pela Universidade Estadual de Londrina (2007). Especialista em Gerência em Serviços de Enfermagem (2009) e Controle de Infecção em Serviços de Saúde (2009). Mestre em Enfermagem Fundamental pela Escola de Enfermagem de Ribeirão Preto EERP/USP (2012).

${ }^{4}$ Graduada em Enfermagem pela Universidade de São Paulo (1989), Especializada em Enfermagem em Estomaterapia pela Escola de
}

na literatura cientifica nacional e internacional, sobre estratégias educacionais para o autocuidado em domicilio de pacientes cirúrgicos, de modo a nortear a assistência perioperatória. Revisão integrativa da literatura, realizada na base de dados LILACS e nas bibliotecas eletrônicas PubMed, DEDALUS e SciELO, com artigos científicos publicados em português, inglês e espanhol entre 2005 e 2016. Foram selecionados quatro

Enfermagem da Universidade de São Paulo (1995). Mestre (1996) e Doutor (2001) em Enfermagem Fundamental pela Escola de Enfermagem de Ribeirão Preto USP. Atualmente é Professor Doutor da Universidade de São Paulo; membro Efetivo da Comissão Assessora de Graduação do Departamento de Enfermagem Geral e Especializada da EERP/USP. Revisor de periódicos CuidArte Enfermagem, Revista Científica das Faculdades Integradas de Jaú, Revista Enfermagem Brasil, Revista Latinoamericana de Enfermagem, Revista Eletrônica de Enfermagem, Revista Texto \& Contexto e Revista Escola Anna Nery Revista de Enfermagem. Pesquisadora do \&quot;Grupo de Estudo da Reabilitação de Pacientes Cirúrgicos Oncológicos\&quot; da Escola de Enfermagem de Ribeirão Preto ? USP e do Grupo de Pesquisa Processo Saúde Doença na Perspectiva Sociocultural da Universidade Federal de Alfenas UNIFAL. Tem experiência na área de Enfermagem, com ênfase em Enfermagem em Estomaterapia, principalmente na área das patologias intestinais, assistência de enfermagem cirúrgica, oncologia, estomaterapia e formação de recursos humanos para a pesquisa em Enfermagem.

${ }^{5}$ Graduada em Enfermagem-Bacharel pela Universidade Estadual do Norte do Paraná-UENP.

${ }^{6}$ Possui graduação em Engenharia Agronômica pela Escola Superior de Agricultura Luiz de Queiroz da Universidade de São Paulo (1971) Mestrado em Estatística e Experimentação Agronômica pela ESALQ/Universidade de São Paulo (1980). Atualmente é professora assistente na Universidade Estadual do Norte do Paraná (UENP), Campus Luiz Meneghel. Atua na área de estatística e experimentação agrícola. Diretora de Pesquisa da Pró-Reitoria de Pesquisa e PósGraduação da UENP. 
artigos científicos que evidenciaram estratégias educacionais para o ensino do autocuidado perioperatório, como computação móvel com módulos educacionais multimídia; acompanhamento telefônico; educação interativa por meio de internet, $\mathrm{CD}-\mathrm{ROM}$ ou DVD, aulas presenciais, aplicação da Teoria do Autocuidado de Orem, reuniões semanais em grupos, folhetos explicativos e educação permanente dos profissionais de saúde. Estas diferentes estratégias promovem o atendimento das necessidades individuais do paciente, favorecendo o aprendizado sobre procedimento cirúrgico e suas consequências, além do desenvolvimento de habilidades para o autocuidado.

Descritores: Autocuidado; Ensino; Tecnologia em saúde; Enfermagem Perioperatória.

\section{Abstract}

The teaching of self-care stands out as an important strategy for coping with the difficulties that permeate the process of postoperative rehabilitation, allowing people to take care of themselves subsidized by information and recommendations elaborated carefully. This article aimed to identify and assess the evidence found in the national and international scientific literature on educational strategies for self-care at home of surgical patients, in order to guide the practice of nursing.Integrative literature review, held in the database LILACS and in electronic libraries PubMed, DEDALUS and SciELO, with scientific articles published in Portuguese, English and Spanish between 2005 to 2016. Four articles were selected that showed educational strategies for the teaching of self-care as mobile computing with perioperative educational multimedia modules; telephone follow-up; interactive education via CD-ROM or DVD, classroom, application of Theory of Self-care by Orem, weekly meetings in groups, explanatory leaflets and continuing education of health professionals. These different strategies promote meeting the needs of the individual patient, encouraging learning about surgical procedure and its consequences, and the development of skills for self-care.

Keywords: Self-care; Teaching; Technology in health; Perioperative Nursing.

\section{Resumen}

La enseñanza del autocuidado se destaca como una importante estrategia para el enfrentamiento de las dificultades que permean el proceso de rehabilitación postoperatoria, posibilitando que las personas cuiden de sí mismas subsidiadas por informaciones $\mathrm{y}$ recomendaciones elaboradas cuidadosamente. Este artículo tuvo como objetivoidentificar y evaluar las pruebas encontradas en la literatura científica nacional e internacional sobre estrategias educativas para el autocuidado en el 
hogar de los pacientes quirúrgicos, con el fin de orientar la asistencia perioperatoria.Revisión de literatura Integrativa, realizado en la base de datos LILACS y en bibliotecas electrónicas PubMed, DEDALUS y SciELO, con artículos científicos publicados en Portugués, Inglés y español entre 2005 a 2016.Se seleccionaron cuatro artículos científicos que demostraron estrategias educativas para la enseñanza del autocuidado como computación móvil con módulos multimedia educativos perioperatoria; seguimiento telefónico; Educación interactiva a través de internet, CDROM o DVD, aula, aplicación de la teoría de autocuidado de Orem,reuniones semanales en grupos, folletos explicativos y formación continuada de los profesionales de la salud. Estas diferentes estrategias promoción las necesidades de cada paciente, fomentar el aprendizaje sobre el procedimiento quirúrgico y sus consecuencias y el desarrollo de habilidades para el autocuidado.

Palabras clave: El cuidado personal; La enseñanza;Tecnología en salud; Enfermería perioperatoria.

\section{Introdução}

Estima-se que anualmente sejam realizadas cerca de 234 milhões de cirurgias em todo mundo,associadas aelevados índices de complicações pós-cirúrgicas, tais como atelectasias pulmonares, casos de trombose venosa profunda, deiscências e infecções da ferida operatória, dentre outras ${ }^{(1-2)}$.

O tratamento cirúrgico pode ser percebido como um evento de risco pelo paciente, assim como pode ocasionar incapacidades físicas, temporárias ou permanentes, repercussões na percepção quanto à imagem corporal,desequilíbrios e fragilidades emocionais, que repercutem negativamente na recuperação pósoperatória $^{(3)}$. Nesse contexto, pode ocorrer comprometimento dos resultados da assistência, bem como acréscimo relevante aoscustos com o tratamento ${ }^{(2)}$.

A necessidade de um procedimento cirúrgico traz inúmeras implicaçõespara o paciente e sua família, sendo fundamental, para o enfrentamento desse desafio, que os mesmos tenham informações claras e consistentes, especialmente no que se refere à continuidade do cuidado após a alta hospitalar $^{(4-5)}$.

A disponibilização de informações sobre o procedimento cirúrgico e suas consequências, facilitaa adaptação do paciente às novas condições e o torna participante na sua preparação e recuperação pós-cirúrgica. Para tanto, faz-se necessária uma assistência planejada, individualizada e humanizada. Nesse sentido, a educação em saúde representa uma ferramenta que contextualiza as realidades sociais, econômicas e culturais dos 
pacientes na promoção da saúde, tratamento e reabilitação ${ }^{(4)}$.

Evidências apontam que o cuidado educacional influencia de forma positiva a evolução pós-operatória, aliviando o estresse dos pacientes submetidos aos procedimentos cirúrgicos $^{(6)} . \mathrm{O}$ processo educacional para $\mathrm{o}$ paciente tem como objetivo promover $\mathrm{o}$ conhecimento e desenvolver suas habilidades, assim como de seus familiares e/ou cuidador, o que garante ações efetivas para manter e melhorar o estado de saúde do indivíduo. Esse processo pode ocorrer por meio da comunicação verbal ou diversos outros recursos educacionais e tecnológicos ${ }^{(7)}$.

$\mathrm{O}$ ensino do autocuidado se destaca como importante estratégia para o enfrentamento das dificuldades que permeiam o processo de reabilitação pós-operatória, possibilitando que as pessoas cuidem de si mesmas subsidiadas por informações e recomendações elaboradas criteriosamente, seja por meio de orientações pessoais ou pela utilização de novas tecnologias online ou $\operatorname{digitais}^{(8)}$.

O enfermeiro, como integrante da equipe assistencial, pode favorecer $\mathrm{o}$ enfrentamento das condições adversas em saúde de pacientes em seu período pósoperatório, mediante a elaboração do planejamento assistencial adequado.

No campo da enfermagem, e com base na Teoria de Dorothea Orem, de 1959, considera-se o autocuidado como "o desempenho ou a prática de atividades que os indivíduos realizam em seu benefício para manter a vida, a saúde e o bemestar"(9). Assim, para o indivíduo que não possui habilidades suficientes para atender a sua demanda de autocuidado, torna-se imprescindível que outra pessoa exerça tais cuidados, no caso, o enfermeiro, um familiar ou cuidador ${ }^{(8)}$.

No que tange à assistência perioperatória, é importante que toda equipe de saúde participe do processo de orientação e motivação do paciente, com vistas a promover a continuidade no tratamento e recuperação pós-operatória, assim como estimular sua participação ativa no autocuidado, principalmente no que se refere à ferida operatória, visando a prevenção decomplicações potenciais ${ }^{(10)}$.

O autocuidado é essencial ao paciente, pois lhe oferece subsídios que o ajudarão a melhorar sua atividade social e sua função familiar, influenciando de forma positiva na adesão ao tratamento e na sua reabilitação ${ }^{(5)}$.

No momento da alta hospitalar, tanto o paciente quanto seus familiares podem desenvolver sentimentos negativos, como a ansiedade e a insegurança, uma vez que se sentem desprotegidos da vigilância constante da equipe de saúde presente no âmbito hospitalar. 
Pesquisas direcionadas para esse tema poderão subsidiar a compreensão das necessidades dos pacientes no período pós-alta cirúrgica, como também nortear a prática da enfermagem no desenvolvimento de ações educativas individualizadas e métodos a serem utilizados ${ }^{(5)}$.

Este estudo visa, identificar e avaliar as evidências encontradas na literatura científica nacional e internacional, sobre estratégias educacionais para o autocuidado em domicilio de pacientes cirúrgicos, de modo a nortear a assistência perioperatória.

\section{Métodos}

Optou-se pela revisão integrativa da literatura com a análise de pesquisas que visam dar suporte à tomada de decisões e à melhoria da prática clínica, possibilitando a síntese do estado do conhecimento de um determinado assunto $^{(11)}$.

O método subsidia a Prática Baseada em Evidências (PBE), voltada ao cuidado clínico e ao ensino fundamentado no conhecimento e na qualidade da evidência. $\mathrm{O}$ processo de elaboração da revisão integrativa compõe-se de seis fases:elaboração da pergunta norteadora; busca na literatura científica; coleta de dados; análise crítica dos estudos incluídos; discussão dos resultados e apresentação da revisão integrativa ${ }^{(12)}$.

O presente estudo ocorreu a partir da seguinte questão norteadora: "O que há publicado, nos últimos 11 anos, sobre estratégias educativas que favoreçam o autocuidado pós-alta hospitalar de pacientes cirúrgicos?”.

Os Descritores em Ciências da Saúde (DeCS), utilizados para busca das publicações no Banco de Dados Bibliográficos da USP (DEDALUS)e biblioteca Literatura Latino Americana do Caribe em Ciências da Saúde (LILACS),foram "autocuidado", "perioperatório" e “educação”. Já, na National Library of Medicine(PubMed), foramempregados os termos do Medical SubjectHeadings (MESH)“careperioperative”, "education" e "self care", e, na biblioteca eletrônicaScientific Eletronic Library Online (SciELO),foram utilizados "assistência perioperatória", “alta hospitalar" ou "autocuidado".

Os critérios de inclusão adotados foram: artigos obtidos na íntegra $\mathrm{e}$ gratuitamente em meios virtuais, que abordassem as estratégias de ensino para facilitar o autocuidado após a alta hospitalar de pacientes cirúrgicos, no período e nos idiomas estabelecidos. Dos resultados obtidos, por meio dessa busca, foram selecionados os artigos que apresentavam nos títulos a temática em questão. Por sua vez, os critérios de exclusão foram: publicações somente em resumo e não disponíveisem periódicos científicos de Enfermagem.Antes da utilização dos critérios de exclusão, foi obtido um total de 
550 artigos, após a filtragem restaram 66 estudos, os quais foram avaliados para compor a amostra do presente estudo.

Após a leitura dos títulos e resumos e em concordância com os critérios de inclusão e exclusão, foram pré-selecionadas 17 publicações. A leitura exaustiva destas, resultou em uma amostra de quatro artigos científicos (Quadro 1), os quais responderam a questão do estudo.

A amostra final foi constituída por artigos que abordaram a utilização de estratégias educativas favoráveis ao autocuidado no período pós-alta hospitalar de pacientes cirúrgicos, indexados na base de dados LILACS e nas bibliotecas eletrônicasPubMed, DEDALUS e naSciELO, publicados em português, inglês e espanhol.

Para categorização dos estudos, as informações foram extraídas utilizando-se um instrumento validado por Ursi ${ }^{(13)}$. Os artigos foram avaliados e os resultados foram sintetizados considerando as especificidades e similaridades do conteúdo.Na avaliação dos artigos, com base na categorização dos estudos, foi focalizado os objetivos, a metodologia, os resultados e a conclusão.

$\mathrm{O}$ instrumento refere-se às categorias temáticas, objetivos do estudo e classificação de seus níveis de evidência. $\mathrm{O}$ instrumento contempla dados de: identificação (título do artigo, título do periódico, autores, país, idioma e ano de publicação); instituição sede do estudo; periódico de publicação; características metodológicas do estudo (tipo de publicação, objetivo, população, amostra, tipo de desenho do estudo, aspectos abordados, categorização do tema, resultados e conclusões e nível de evidência); base de dados de acesso e fonte de obtenção de publicação na íntegra $^{(13)}$.

Com a interpretação e discussão das evidências cientificas, foi possível identificar as estratégias educativas que auxiliam no ensino do autocuidado após a alta hospitalar. Espera-se que os resultados deste estudo contribuam para nortear as práticas assistenciais, principalmente no que se refere aos profissionais deenfermagem, sobre as principais metodologias utilizadas para o ensino dos pacientes cirúrgicos.

\section{Resultados}

A amostra deste estudo é composta por quatro artigos selecionados, a serem: na DEDALUS (1), na LILACS (1), na SciElo(1) e naPubMed (2), sendo que dois artigos iguais foram encontrados na DEDALUS e na LILACS.Em relação aos idiomas dos quatroestudos selecionados,dois artigos foram publicados em inglês e dois em língua portuguesa.

Quadro 1: Demanda de aprendizagem de pacientes cirúrgicos e estratégias de ensino segundo amostra do estudo. Bandeirantes, Paraná, 2016 


\begin{tabular}{|c|c|c|c|c|c|}
\hline Temática & Artigo & Título & Autor(es) & Periódico/Ano & Estratégias utilizadas \\
\hline \multirow{4}{*}{$\begin{array}{c}\text { Métodos de } \\
\text { ensino para o } \\
\text { autocuidado em } \\
\text { domicilio de } \\
\text { pacientes } \\
\text { cirúrgicos }\end{array}$} & 1 & $\begin{array}{l}\text { Patient Education } \\
\text { Self-Management } \\
\text { During Surgical } \\
\text { Recovery: } \\
\text { Combining Mobile } \\
\text { (iPad) and a Content } \\
\text { Management System }\end{array}$ & Cook DJ et al. & $\begin{array}{l}\text { TELEMEDICINE and } \\
\text { HEALTH. } 2014\end{array}$ & $\begin{array}{l}\text { Plataforma eletrônica por meio de } \\
\text { dispositivos móveis(tablets); módulos } \\
\text { multimídia. }\end{array}$ \\
\hline & 2 & $\begin{array}{l}\text { Estratégias de ensino } \\
\text { para o autocuidado de } \\
\text { estomizados } \\
\text { intestinais }\end{array}$ & Silva JS et al. & Rev Rene. 2014 & $\begin{array}{l}\text { Programas multimídias, } \\
\text { acompanhamento telefônico, educação } \\
\text { interativa por meio de CD-ROM e DVD, } \\
\text { educação permanente, ensino em } \\
\text { domicilio, aula presencial e aplicação da } \\
\text { Teoria do Autocuidado de Orem. }\end{array}$ \\
\hline & 3 & $\begin{array}{l}\text { Visibilidade do } \\
\text { projeto de extensão } \\
\text { para promoção da } \\
\text { assistência } \\
\text { perioperatória: ponto } \\
\text { de vista } \\
\text { multidisciplinar }\end{array}$ & $\begin{array}{l}\text { Souza NVDO } \\
\text { et al. }\end{array}$ & $\begin{array}{l}\text { Esc Anna Nery Enferm. } \\
2008\end{array}$ & $\begin{array}{l}\text { Reuniões em grupo com orientações } \\
\text { individuais, álbum seriado e folheto } \\
\text { educativo. }\end{array}$ \\
\hline & 4 & $\begin{array}{l}\text { The development of } \\
\text { an evidence-based } \\
\text { patient booklet } \\
\text { for patients } \\
\text { undergoing lumbar } \\
\text { discectomy } \\
\text { and un-instrumented } \\
\text { decompression }\end{array}$ & $\begin{array}{l}\text { McGregor } \\
\text { AH et al. }\end{array}$ & EurSpine J. 2007 & Cartilha educativa e aconselhamento. \\
\hline
\end{tabular}

O estudo 1 se refere à apresentação de uma nova plataforma de saúde eletrônica, por meio de dispositivos móveis, contendo um sistema de gerenciamento de conteúdo que objetivou fornecer uma nova abordagem tecnológica, uma capacitação efetiva para os pacientes idosos em pós-operatório de cirurgia cardíaca. Foram fornecidos tablets contendo uma lista de cuidados diários para cada participante do estudo ${ }^{(14)}$.

A rede de dados apresentava-se conectada a um painel de controle sem fio, Rev. Gestão \& Saúde (Brasília) Edição Especial, fev. 2019. onde eram agregados e exibidos para os provedores. O programa apresentava um plano de internação hospitalar, onde os pacientes se envolviam com a autoaprendizagem por meio de módulos educacionais multimídia para o dia-a-dia, com as principais dúvidas que esses pacientes pudessem apresentar ${ }^{(14)}$.

A população de estudo consistiu em pacientes com idade superior a 50 anos, com ausência de déficit cognitivo em seu registro médico e com uma previsão de internação entre 5-7 dias para a realização de cirurgia 
cardíaca eletiva. A tecnologia móvel, mesmo que desconhecida para muitos pacientes com idade mais avançada, pode ser usada em prol da saúde, com gerenciamento de conteúdo sobre sua condição de saúde para o autocuidado de pacientes cirúrgicos, e- cria uma maneira simples de manter o conteúdo atualizado e consistente ${ }^{(14)}$.

O estudo 2 sintetizou estratégias de ensino para pacientes estomizados intestinais, sendo que as estratégias de aprendizagem para o autocuidado domiciliar indicadas, foram programa multimídia de ensino, sendo o mais citado dos estudos acompanhamento telefônico por enfermeiros especialistas, para o fornecimento de informações e orientações aos pacientes; ensino de paciente e família no domicilio $^{(15)}$.

Ainda nesse estudo, foram utilizados educação interativa, por meio da internet ou multimídias como CD-ROM e DVD combinando modos de informação; aula presencial (tanto para o paciente, familiar e profissional da saúde); programa de educação permanente para os profissionais da Atenção Primária e Secundária, e aplicação da Teoria do Autocuidado de Orem ${ }^{(15)}$.

Porém, parte deste estudo se mostrou limitada, por não demonstrar a efetividade da utilização de estratégias de ensino por meio das bases digitais, em relação ao ensino do autocuidado, de forma tradicional, enfermeiropaciente. Entretanto, as estratégias pesquisadas indicaram a viabilidade destas alternativas no seguimento de pacientes cirúrgicos pós-alta hospitalar, contribuindo para a qualidade da assistência prestada ${ }^{(15)}$.

O estudo 3 identificou o conhecimento de uma equipe multidisciplinar acerca das atividades desenvolvidas pelos membros de um projeto de extensão intitulado "Orientando o cliente em situação cirúrgica para diferenciar o cuidado" de uma universidade pública. As orientações fornecidas eram sobre a dinâmica do processo de internação, desmistificação da experiência cirúrgica para a redução das alterações biopsíquicas e sociais decorrentes ao procedimento cirúrgico ${ }^{(16)}$.

As orientações ocorreram de duas formas: reuniões semanais, com grupos de pacientes no período pré-operatório, e orientações individuais para alta hospitalar, com esclarecimentos para o autocuidado no domicilio $^{(16)}$.

A primeira atividade consistiu em uma explanação problematizada, utilizando álbum seriado com gravuras referentes às situações do perioperatório e fotos do ambiente do Centro Cirúrgico. Estas estratégias instrucionais possibilitaram maior aproximação com o contexto desconhecido do perioperatório para diminuir os níveis de ansiedade $^{(16)}$.

E a segunda atividade foi $\mathrm{o}$ fornecimento de orientações individuais que capacitem o paciente e/ou familiar para a o 
autocuidado domiciliar, assim como limitadas, imprecisas ou inconsistentes. Os minimizar reações emocionais desagradáveis decorrentes da alta hospitalar. Foram utilizados folhetos explicativos sobre cuidado com a ferida operatória e realização do curativo, alimentação, retorno da atividade sexual e produtiva, orientações acerca de possíveis complicações, além das manifestações clínicas decorrentes de um procedimento cirúrgico ${ }^{(16)}$.

O estudo aponta as estratégias contidas no projeto como uma maneira eficaz de promover orientações para o cuidado em saúde, podendo ser por meio da formação de grupos que contam com pessoas de interesses comuns ou que se encontram em condições semelhantes ${ }^{(16)}$.

No estudo 4, foi elaborada uma cartilha educativa centrada no paciente, sobre informações e conselhos para reabilitação pósoperatória, para avaliação e validação. A finalidade da cartilha era reduzir a incerteza, promover crenças potenciais, incentivar a reabilitação precoce e fornecer conselhos práticos sobre o autocuidado ${ }^{(17)}$.

$\mathrm{Na}$ avaliação e validação participaram 14 pacientes $(70 \%)$ dos 20 pacientes para os quais foi enviada a cartilha. Todos os 14 pacientes consideraram o material de fácil leitura, claro e interessante. Nesse contexto, evidenciou-se que orientações pós-operatórias não devem se deter somente ao fornecimento de orientações verbais, pois elas podem ser materiais impressos são um método simples, de grande potencialidade, barato e popular, de prestação de informações que podem melhorar a adesão, aliviar a ansiedade, promover o bem estar psicológico e reduzir as alegações de negligência sobre profissionais da saúde $\mathrm{e}^{(17)}$.

\section{Discussão}

A análise dos estudos sobre métodos de ensino para o autocuidado de pacientes cirúrgicos, reforça a importância do papel dos profissionais da equipe de saúde em fornecer informações que auxiliem na prevenção e controle de complicações, contribuindo positivamente com sua recuperação. Nas últimas décadas, usuários dos serviços de saúde vêm se tornando mais conscientes e participativos no que se refere ao seu tratamento e recuperação, fato que evidencia a necessidade de estratégias de educaçãopara garantir a manutenção e promoção da saúde ${ }^{(18)}$. O foco da educação em saúde é baseado na independência da pessoa, a qual deverá aprender a lidar com suas fragilidades, adaptando-se à próprias necessidades e preservando sua qualidade de vida ${ }^{(19)}$.

No decorrer dos anos, a sociedadevem passando por transformações em conjunto com os avanços tecnológicos inseridos no cotidiano da população. Equipamentos cada vez mais modernos e redes que viabilizam a comunicação entre as pessoas, como 
computadores, tablets, telefones e a própria rede global de computadores (internet) têmfacilitado a vida das pessoas e tornaram-se parte da cultura social ${ }^{(7)}$.

As ferramentas tecnológicas possibilitama obtençãode informação em tempo real, impactando na educação do paciente, e por meiodessas ferramentas, indivíduos, famílias e comunidades assumem um papel ativo no que concerne aoscuidados com a própria saúde, visto que as tecnologias agregam novos conhecimentos para suas $\operatorname{vidas}^{(7)}$.

Um estudo recente verificou que as mídias estão sendo utilizadas com frequência para a educação em saúde, dentre elas: programas multimídias, vídeos, CD-ROM, Websites, videoconferência, fita de áudio, radio, televisão, dentre outras. Essa referência corrobora com os resultados alcançados no presente estudo, comprovando que, cada vez mais, programas tecnológicos estão sendo usados em prol da educação em saúde, de modo a tornar os indivíduos mais participativos com o aumento de seu envolvimento no processo de tomada de decisão em relação à sua saúde ${ }^{(19)}$.

Porém, para a criação de materiais educativos eficientes, é indispensável que se conheça arealidade e as expectativas dos pacientes, priorizando as suas necessidades e não somente as exigências terapêuticas ${ }^{(18-20)}$.
O sucesso das ações educativas só será alcançado a partir do conhecimento da população usuária, suas rotinas, crenças e condições em que estão inseridas, e ainda se faz necessário o envolvimento dos pacientes nas atividades de cuidado e não a imposição do conhecimento. Essas; representam medidas imprescindíveis às ações educativas verdadeiramente efetivas $^{(18-20)}$.

Para o uso de tecnologias educacionais, o educador deve avaliar o público alvo, considerando suas peculiaridades cognitivas e a forma de apresentação do conteúdo.Os materiais educativos devem ter como objetivofacilitar o trabalho dos profissionais de saúde na comunicação e orientação de pacientes e familiares. Porém, jamais substituir a orientação verbal e sim asubsidiar ${ }^{(21)}$.

Também, se faz necessário que os profissionais realizem orientações claras e objetivas, sem ambiguidades, que sejam transmitidas por meio de tecnologias educacionais capazes de mobilizarem a atenção e motivar sua utilização. Os enfermeiros podem ajudar os pacientes e suas famílias a aprenderem como manter a saúde, como restaurá-la ou como fazer adaptações para sua maiorindependência ${ }^{(4)}$.

Os pacientes, ao retornarem aos seus domicílios, se deparam com situações desafiadoras, sendo nesse ambiente que começam a surgir as dúvidas e mudanças de hábitos, alterando suasrotinas.Portanto, é 
importante que se valorize as dúvidas,por mais simples que sejam, assim comoos esclarecimentos, para que o paciente possa dar continuidade a sua recuperação no domicilio $^{(22)}$.

Em um estudo realizado em 2015, com pacientes oncológicos e seus familiares e/ou cuidadores, constatou-se ser possível ajudá-los por meio de folheto educativo, pois este material de ensino os auxiliou a nortear suas ações de cuidado em domicílio, sendo útil, principalmente, nos processos decisórios tanto sob o ponto de vista do cuidador como do paciente $^{(23)}$.

A educação em saúde é uma prática inerente da enfermagem. O folheto educativo é um material impresso querepresenta uma estratégia acessível e eficiente para a promoção do autocuidado, porém, vale ressaltar que a contribuição deste tipo de recurso somente será efetiva mediante a real compreensão do leitor ${ }^{(24)}$.

A cartilha, como estratégia educacional apresentada nos resultados, é um material educativo potencial, que visapossibilitar ao paciente melhor compreensão acerca da sua enfermidade, favorecendo a reflexão para o desenvolvimento da sua capacidade de autonomia para o cuidadopós-alta hospitalar $^{(21)}$.

Autores corroboram que o compartilhamento de informações entre equipe, paciente e familiar, é considerado um meio de construção do conhecimento, que irá auxiliar pacientes e familiaresà aprendizagem de cuidados pós-alta hospitalar ${ }^{(18,22)}$.

A partir desse entendimento, a sistematização da educação para esta clientela,utilizandoferramentas tecnológicas,visuais, impressas, auditivas e lúdicas, é um dos processos essenciais para a promoção da saúde, pois a capacitapara a recuperação e para areabilitação,com reflexos positivos no estado de saúde e na qualidade de $\operatorname{vida}^{(18,25)}$.

Em diversas instituições, as orientações para a continuidade do cuidado pós-alta é deficiente, em decorrência do baixo número de profissionais, do despreparo da equipe quanto aos recursos e métodos de ensino, falta de embasamento teórico, aprimoramento, treinamento e habilidades, principalmente por parte da equipe de enfermagem, apesar de serem os profissionais em contato constante e direto com os pacientes e seus familiares ${ }^{(25)}$.

Os profissionais devem estar aptos a fornecer informações por meio de estratégias adequadas, de forma compreensível, clara e com linguagem adequada ao paciente. Ressalta-se, também, que as orientações devem ser realizadas de forma gradual para evitar a saturação do paciente. Entretanto, fica claro que o enfermeiro deve combinar estratégias de ensino adequadas com as habilidades cognitivas de cada paciente, considerando que são pessoas com níveis de 
Revista Eletrônica Gestão \& Saúde ISSN: 1982-4785

escolaridade, dúvidas e dificuldades distintas sobre o autocuidado após a alta hospitalar ${ }^{(25)}$.

\section{Conclusão}

Ficou evidente a escassez de estudos sobre estratégias educacionais para $\mathrm{o}$ autocuidado de pacientes cirúrgicos, e também apresentou limitações em relação à efetividade e consolidação dessas estratégias. Apesar de todos os estudos selecionados apresentarem aspectos importantes à prática clínica em Enfermagem Perioperatória, há necessidade de mais pesquisas sobre estratégias educacionais e métodos de ensino para o autocuidado em domicilio.

Dentre as estratégias mais citadas estão as que utilizam tecnologias como programas multimídias, plataformas eletrônicas com uso de tablets, educação interativa por meio da Internet, CD-ROM， DVD， álbum seriadoeacompanhamento telefônico. Isso reflete a globalização que apopulação vivencia, sendo que,cada dia mais, as tecnologias estão presentes no cotidiano de profissionais e pacientes. Tal realidade evidencia a necessidade da atualização e emprego de saberes e tecnologias pelos profissionais da saúde em prol da promoção e reabilitação da saúde humana.

Entretanto, deve-se levar em conta que algumas estratégias tecnológicas envolvem maior recurso tecnológico e, portanto, acabam não sendo viáveis para muitas instituições que
Estratégias para o Ensino do Autocuidado...

trabalham com recursos limitados. Por outro lado, deve-se considerar também, aspectos culturais e socioeconômicos que tendem a interferir negativamentenos resultados esperados. Nesse sentido, este estudo também resgatoua possibilidade do emprego de tradicionais materiais impressos em formato de folheto educativo e de cartilhas,que podem auxiliar o paciente a aumentar sua autonomia e segurança no autocuidado domiciliar.

Apesar de todos os métodos apresentados para orientar pacientes cirúrgicos a se cuidarem em seus domicílios, os estudos não omitirama importância da comunicação verbal e de orientações individualizadas entre profissional e paciente como forma de ensino presencial.

De modo geral, as estratégias de ensino apresentadas nos estudos, apontam à viabilidade deseguimento dos pacientes cirúrgicos após a alta hospitalar, de forma a contribuir para sua segurança eagilização do processo de reabilitação. 
Revista Eletrônica Gestão \& Saúde ISSN: 1982-4785

Stadler DV, Giordani AT, Paulino GME et al

\section{Referências}

1. de Brito Paranaguá TT, Bezerra ALQ, de Camargo AEB, de Azevedo Filho SFM. Prevalence of no harm incidents and adverse events in a surgical clinic. Acta Paul Enferm. 2013;26(3):256-62.

2. Santana RF, do Amaral DM, Pereira SK, Delphino TM, Cassiano KM. Ocorrência do diagnóstico de enfermagem de recuperação cirúrgica retardada entre adultos e idosos. Acta Paul Enferm. 2014;27(1):35-9.

3. Perrando M, Beuter M, Brondani CM, Roso CC, dos Santos TM, Predebon GR. O preparo pré-operatório na ótica do paciente cirúrgico. Revista de Enfermagem da UFSM. 2011;1(1):61-70.

4. Castro AP, Oikawa SE, Domingues TAM, Hortense FTP, De Domenico EBL. Educação em Saúde na Atenção ao Paciente Traqueostomizado: Percepção de Profissionais de Enfermagem e Cuidadores. Revista Brasileira de Cancerologia. 2014;60(4):305-13.

5. Romanzini AE, Jesus APMd, Sasaki VDM, Damiano VB, Gomes JJ. Orientações de enfermagem aos pacientes sobre o autocuidado e os sinais e sintomas de infecção de sítio cirúrgico para a pós-alta hospitalar de cirurgia cardíaca reconstrutora. Revista Mineira de Enfermagem. 2010;14(2):239-43.

6. Giordani AT, Stadler DV, Paulino GME, Zanardo RR, Sonobe HM, Valerio MA. Demand of hospitalized surgical patients information: a descriptive and prospective study. Online braz $\mathrm{j}$ nurs. 2016;15(2):124-33.

7. Bastos BG, Ferrari DV. Internet e Educação ao paciente. Arq. Int. Otorrinolaringol. São Paulo. 2011; 15(4):515-522

8. Costa SRDd, Castro EABd, Acioli S. Capacidade de autocuidado de adultos e idosos hospitalizados: implicações para o cuidado de enfermagem*. Revista Mineira de Enfermagem. 2013;17(1):193-207.

9. Foster PC, Bennett AM. Dorothea E. Orem. In: George JB. Teorias de enfermagem: os fundamentos para a prática profissional. $4^{\mathrm{a}} \mathrm{ed}$. Porto Alegre: Artes Médicas; 2000. p. 83-102.

10. Santos JBdos, Porto SG, Suzuki LM, Sostizzo LZ, Antoniazzi JL. Avaliação e tratamento de feridas: orientações aos profissionais de saúde. Hospital das
Estratégias para o Ensino do Autocuidado...

clínicas de Porto Alegre. 2011. Dísponível em: http://hdl.handle.net/10183/34755.

11. Mendes KDS, Silveira RCdCP, Galvão CM. Revisão integrativa: método de pesquisa para a incorporação de evidências na saúde e na enfermagem. Texto and Contexto Enfermagem. 2008;17(4):758.

12. de Souza MT, da Silva MD, de Carvalho R. Revisão integrativa: o que é e como fazer. Einstein (São Paulo). 2010;8:102-6.

13. URSI, Elizabeth Silva. Prevenção de lesões de pele no perioperatório: revisão integrativa da literatura. [dissertação]. Ribeirão Preto: Universidade de São Paulo, Escola de Enfermagem de Ribeirão Preto; 2005.

14. Cook DJ, Moradkhani A, Douglas KSV, Prinsen SK, Fischer EN, Schroeder DR. Patint education selfmanagement during surgical recovery: cominingmóbile (iPad) an a content management system. Telemedical and e-health. 2014; 20(4):312-17.

15. da Silva J, Sonobe HM, Buetto LS, dos Santos MG, de Lima MS, sasaki VDM. Estratégias de ensino para o autocuidado de estomizados intestinais. Rev Rene. 2014; 15(1): 166-73.

16. Souza NVDO, Silva MF, Nunes KSM, Assumpção LR, Morgado FM, Amorin LKA. Visibilidade do projeto de extensão para a promoção da assistência perioperatória: ponto de vista multidisciplinar. Esc Anna Nery RevEnferm. 2008: 12(2): 329-33.

17. McGregor AH, Barton AK, Sell P, Waddell G. The development of an evidence-based patient booklet for patients undergoing lumbar discectomy and uminstrumented decompression. EurSpine J. 2007; 16: 339-46.

18. Meirelles ARN, Menezes D, Sena MHLG, Cruz IS, Luedy A, Ribeiro Junior HC. O papel da educação de pacientes e familiares na construção de um processo de segurança e qualidade em um Hospital Universitário. Revista Acred. 2013; 3(5): 23-33.

19. Grossi MG, Maruxo HB, Kobayashi RM, Prado C, Heiman C. Revisão Integrativa: A Utilização das Mídias Interativas para Educação em saúde.

20. Gozzo TO, Lopes RR, Prado MAS, da Cruz LAP, de Almeida AM. Informações para a elaboração de um 
Revista Eletrônica Gestão \& Saúde ISSN: 1982-4785 Stadler DV, Giordani AT, Paulino GME et al

manual educativo destinado às mulheres com câncer de mama. Esc Anna Nery. 2012; 16(2); 306-11.

21. Áfio ACE, Balbino AC, Alves MDS, Carvalho LVde, Santos MCL, Oliveira NR. Análise do conceito de tecnologia educacional em enfermagem aplicada ao paciente. Rev. Rene.2014; 15(1):158-65.

22. Chahin TTH, Mostardeiro SCTS, Terra MG, da Silva CT, Maciel FB, Soccol KLS. Alta hospitalar do paciente cirúrgico ambulatorial: percepção da equipe de enfermagem na educação em saúde. J. Nurs Health. 2013; 3(1): 115-25.

23. Nascimento EA, Tarcia RML, Magalhães LP, Soares MAL, Suriano MLF, de Domenico EBL. Folhetos educativos em saúde: estudo de recepção. RevEscEnferm USP. 2015;49(3);435-42.

24. Freitas AAS, Cabral IE. O cuidado à pessoa traqueostomizada: análise de um folheto educativo. Esc Anna Nery RevEnferm. 2008; 12(1):84-9.

25. Lenza NFB, Sonobe HM, Buetto LS, Santos MG, Lima MS. The teaching of self-care to ostomy patients and their families: an integrative review. RerBrasPromoç Saúde. 2013; 26(1):139-45.
Estratégias para o Ensino do Autocuidado...

\section{Participação dos autores:}

STADLER, DV procedeu à coleta, organização dos dados, revisão literária e redação do artigo.

GIORDANI, AT orientou e contribuiu com a análise e interpretação dos dados, redação do artigo.

PAULINO, GME acompanhou a construção do artigo e auxiliou nas revisões.

SONOBE, HM acompanhou a construção do artigo e auxiliou nas revisões.

ZANARDO, RR auxiliou na coleta, organização dos dados, redação do artigo e do folheto educativo.

VALÉRIO, MA auxiliou nas revisões.

Recebido: 23.02.2017

Revisado: 12.04 .2017

Aprovado: 25.05.2017 